\title{
USCO SELECTIONS OF DENSELY DEFINED SET-VALUED MAPPINGS
}

\author{
Warren B. MoOrs and Sivajah Somasundaram
}

A set-valued mapping $\Phi: X \rightarrow 2^{Y}$ acting between topological spaces $X$ and $Y$ is said to be "lower demicontinuous" if the interior of the closure of the set $\Phi^{-1}(V):=$ $\{x \in X: \Phi(x) \cap V \neq \emptyset\}$ is dense in the closure of $\Phi^{-1}(V)$ for each open set $V$ in $Y$. Coban, Kenderov and Revalski (1994) showed that for every densely defined lower demicontinuous mapping $\Phi$ acting from a Baire space $X$ into subsets of a monotonely Cech-complete space $Y$, there exist a dense and $G_{\delta}$ subset $X_{1} \subseteq X$ and an usco mapping $G: X_{1} \rightarrow 2^{Y}$ such that $G(x) \subseteq \Phi^{*}(x)$, for every $x \in X_{1}$, where the mapping $\Phi^{*}: X \rightarrow 2^{Y}$ is the extension of $\Phi$ defined by,

$$
\Phi^{*}(x):=\bigcap\{\overline{\Phi(W)}: W \text { is a neighbourhood of } x\} .
$$

In this paper we present a proof of the above result with the notion of monotone Cechcompleteness replaced by the weaker notion of partition completeness. In addition, we observe that if the range space also lies in Stegall's class then we may assume that the mapping $G$ is single-valued on $X_{1}$.

\section{INTRODUCTION}

Selection theorems provide conditions under which there exists a continuous selection for a set-valued mapping. In a recent paper [4], on selection theorems the authors presented a selection theorem for quasi-lower semicontinuous mappings that map from Baire spaces into subsets of topological spaces that are fragmented by complete metrics. In this paper we improve this result by presenting a selection theorem for "lower demicontinuous" mappings that map from Baire spaces into partition complete spaces. Specifically, we show that for a lower demicontinuous mapping $\Phi$ with closed graph acting from a Baire space $X$ into a partition complete space $Y$ there exist a dense and $G_{\delta}$ subset $X_{1} \subseteq X$ and an usco mapping $G: X_{1} \rightarrow 2^{Y}$ such that $G(x) \subseteq \Phi(x)$ for all $x \in X_{1}$. In addition we show that if the range space $Y$ is partition complete and lies in Stegall's class then the mapping $G$ may also be assumed to be single-valued on $X_{1}$. We also show that if the domain space $X$ is $\alpha$-favourable and the range space is partition complete and

Received 24th September, 2001.

Copyright Clearance Centre, Inc. Serial-fee code: 0004-9727/02 \$A2.00+0.00. 
belongs to the class of weakly Stegall spaces then the mapping $G$ is single-valued on an everywhere second category subset of $X$.

We end this section by giving some definitions, then in Section 2 we present the main result and finally in Section 3 we give some applications of our selection theorem.

Let $(Y, \tau)$ be a regular topological space, endowed with a pseudo-metric $d$. A filterbase $\mathcal{F}$ on $Y$ is said to be $d$-Cauchy if for each $\varepsilon>0$ there exists an $F \in \mathcal{F}$ such that $d-\operatorname{diam}(F)<\varepsilon$ and the space itself is said to be partition complete if the pseudo-metric $d$ satisfies the following properties:

(i) every $d$-Cauchy filter-base $\mathcal{F}$ on $Y$ has a $\tau$-cluster point in $Y$ (that is, $\bigcap\{\bar{F}: F \in \mathcal{F}\} \neq \emptyset)$;

(ii) $Y$ is "fragmented" by $d$, that is, for every $\varepsilon>0$ and every non-empty subset $A$ of $Y$ there exists a $\tau$-open subset $B$ of $Y$ such that $A \cap B \neq \emptyset$ and $d-\operatorname{diam}(A \cap B)<\varepsilon$.

(Note: It follows from (i) that in a partition complete space $\bigcap\{\bar{F}: F \in \mathcal{F}\}$ is nonempty and compact for every $d$-Cauchy filter-base $\mathcal{F}$.) The class of partition complete spaces is quite large including all the Cech complete spaces. More details on partition completeness can be found in [5].

\section{Selection Theorem}

Let $\Phi: X \rightarrow 2^{Y}$ be a set-valued mapping acting from a topological space $X$ into subsets of a topological space $Y$. We call the mapping $\Phi$ lower demicontinuous on $X$ if for every open set $V$ in $Y$, the interior of the closure of the set $\Phi^{-1}(V):=\{x \in X:$ $\Phi(x) \cap V \neq \emptyset\}$ is dense in the closure of $\Phi^{-1}(V)$, that is, $\operatorname{int}\left(\overline{\Phi^{-1}(V)}\right)$ is dense in $\overline{\Phi^{-1}(V)}$. When $\{x \in X: \Phi(x) \neq \emptyset\}$ is dense in $X$, we say $\Phi$ is densely defined.

Lemma 1. Consider a lower demicontinuous mapping $\Phi$ from a topological space $X$ into subsets of a topological space $Y$. For each pair of non-empty open sets $U$ in $X$ and $V$ in $Y$, the mapping $\Phi_{(U, V)}$ from $U$ into subsets of $V$ defined by, $\Phi_{(U, V)}(x):=\Phi(x) \cap V$ is a lower demicontinuous mapping on $U$.

ProOF: The proof of the lemma follows from the fact that for each open set $W \subseteq V$, $\Phi_{(U, V)}^{-1}(W)=\Phi^{-1}(W) \cap U$.

A set-valued mapping $\Phi: X \rightarrow 2^{Y}$ acting between topological spaces $X$ and $Y$ is said to be an usco mapping if for each $x \in X, \Phi(x)$ is a non-empty compact subset of $Y$ and for each open set $W$ in $Y,\{x \in X: \Phi(x) \subseteq W\}$ is open in $X$.

THEOREM 1. Let $X$ be a Baire space and $Y$ be a Hausdorff partition complete space and let $\Phi$ be a densely defined lower demicontinuous set-valued mapping acting from $X$ into subsets of $Y$. Then there exist a dense and $G_{\delta}$-subset $X_{1} \subseteq X$ and an usco mapping $G: X_{1} \rightarrow 2^{Y}$ with $G(x) \subseteq \Phi^{*}(x)$ for all $x \in X_{1}$, where the mapping 
$\Phi^{*}: X \rightarrow 2^{Y}$ is defined by,

$$
\Phi^{*}(x):=\bigcap\{\overline{\Phi(W)}: W \text { is a neighbourhood of } x\}
$$

In particular, $\left\{x \in X: \Phi^{*}(x) \neq \emptyset\right\}$ is residual in $X$.

Proof: Let $d$ be the fragmenting pseudo-metric on $Y$ associated with the partition completeness of $Y$. To prove our theorem we inductively construct a sequence of families of ordered pairs $\mathcal{F}^{n}:=\left\{\left(U_{\alpha}^{n}, \Phi_{\alpha}^{n}\right): \alpha \in \Lambda^{n}\right\}$ consisting of non-empty open subsets $\left\{U_{\alpha}^{n}: \alpha \in \Lambda^{n}\right\}$ of $X$ and densely defined lower demicontinuous mappings $\left\{\Phi_{\alpha}^{n}: \alpha \in \Lambda^{n}\right\}$ such that for each $\alpha \in \Lambda^{n}, \Phi_{\alpha}^{n}$ maps $U_{\alpha}^{n}$ into subsets of $Y$.

BASE STEP. Consider $\Lambda^{0}:=\{\emptyset\}, U_{\emptyset}^{0}:=X$ and $\Phi_{\emptyset}^{0}:=\Phi$ and define,

$$
\mathcal{F}^{0}:=\left\{\left(U_{\alpha}^{0}, \Phi_{\alpha}^{0}\right): \alpha \in \Lambda^{0}\right\} \text { and } W^{0}:=\bigcup\left\{U_{\alpha}^{0}: \alpha \in \Lambda^{0}\right\}=X
$$

For each $n \in \mathbb{N}$, we require the family $\mathcal{F}^{n}$ to have the following properties:

$\left(a_{n}\right) \quad U_{\alpha}^{n} \cap U_{\beta}^{n}=\emptyset$ for each $\alpha \neq \beta, \alpha, \beta \in \Lambda^{n}$;

$\left(b_{n}\right) \quad W^{n}:=\bigcup\left\{U_{\alpha}^{n}: \alpha \in \Lambda^{n}\right\}$ is dense in $X$;

$\left(c_{n}\right) d-\operatorname{diam}\left[\Phi_{\alpha}^{n}\left(U_{\alpha}^{n}\right)\right]<1 / n$ for each $\alpha \in \Lambda^{n}$;

$\left(d_{n}\right)$ for each $\alpha \in \Lambda^{n}$ there exists a $\beta \in \Lambda^{n-1}$ such that $U_{\alpha}^{n} \subseteq U_{\beta}^{n-1}$ and $\Phi_{\alpha}^{n}(x) \subseteq$ $\Phi_{\beta}^{n-1}(x)$ for each $x \in U_{\alpha}^{n}$.

STEP 1. Consider $\mathcal{F}^{\mathbf{l}}:=\left\{\left(U_{\alpha}^{\mathbf{l}}, \Phi_{\alpha}^{1}\right): \alpha \in \Lambda^{1}\right\}$ a family of ordered pairs satisfying the properties $\left(a_{1}\right),\left(c_{1}\right)$ and $\left(d_{1}\right)$ which is maximal with respect to set inclusion. By Zorn's lemma such a maximal family exists. We shall show that $\mathcal{F}^{\mathbf{l}}$ satisfies property $\left(b_{1}\right)$. If $W^{1}:=\bigcup\left\{U_{\alpha}^{1}: \alpha \in \Lambda^{1}\right\}$ is not dense in $X$ then there exists a non-empty open subset $U$ of $X$ such that $W^{1} \cap U=\emptyset$. Since $Y$ is fragmented by $d$ and $\Phi_{\emptyset}^{0}$ is densely defined there exists an open set $V$ in $Y$ such that $\Phi_{\emptyset}^{0}(U) \cap V \neq \emptyset$ and $d-\operatorname{diam}\left[\Phi_{\emptyset}^{0}(U) \cap V\right]<1$. By the lower demicontinuity of $\Phi_{\emptyset}^{0}$ on $U$ there exists a non-empty open subset $U^{\prime}$ of $U$ such that $\left(\Phi_{\emptyset}^{0}\right)_{\left(U^{\prime}, V\right)}$ is densely defined and lower demicontinuous on $U^{\prime}$ (by Lemma 1). Now $\left(U^{\prime},\left(\Phi_{\emptyset}^{0}\right)_{\left(U^{\prime}, V\right)}\right) \notin \mathcal{F}^{1}$ and $\left\{\left(U^{\prime},\left(\Phi_{\emptyset}^{0}\right)_{\left(U^{\prime}, V\right)}\right)\right\} \cup \mathcal{F}^{1}$ is a family satisfying the properties $\left(a_{1}\right),\left(c_{1}\right)$ and $\left(d_{1}\right)$. This contradicts the maximality of $\mathcal{F}^{1}$ and hence we may conclude that $\mathcal{F}^{\mathbf{l}}$ satisfies property $\left(b_{1}\right)$.

Assuming that we have constructed the families $\mathcal{F}^{k}$ in the sequence satisfying the properties $\left(a_{k}\right),\left(b_{k}\right),\left(c_{k}\right)$ and $\left(d_{k}\right)$ up to and including the $n$th step, we proceed to construct the next step.

STEP $(n+1)$. Consider $\mathcal{F}^{n+1}:=\left\{\left(U_{\alpha}^{n+1}, \Phi_{\alpha}^{n+1}\right): \alpha \in \Lambda^{n+1}\right\}$ a family of ordered pairs satisfying the properties $\left(a_{n+1}\right),\left(c_{n+1}\right)$ and $\left(d_{n+1}\right)$ which is maximal with respect to set inclusion. We shall show that $\mathcal{F}^{n+1}$ satisfies property $\left(b_{n+1}\right)$. If $W^{n+1}:=\bigcup\left\{U_{\alpha}^{n+1}: \alpha \in\right.$ $\Lambda^{n+1}$ \} is not dense in $X$ then there exists a non-empty open subset $U$ of $X$ such that $W^{n+1} \cap U=\emptyset$. Since $W^{n}$ is dense in $X, W^{n} \cap U \neq \emptyset$ and so we may assume that $U \subseteq U_{\beta}^{n}$ 
for some $\beta \in \Lambda^{n}$. Now since $Y$ is fragmented by $d$ and $\Phi_{\beta}^{n}$ is densely defined there exists an open set $V$ in $Y$ such that $\Phi_{\beta}^{n}(U) \cap V \neq \emptyset$ and $d-\operatorname{diam}\left[\Phi_{\beta}^{n}(U) \cap V\right]<1 /(n+1)$. By the lower demicontinuity of $\Phi_{\beta}^{n}$ on $U_{\beta}^{n}$ there exists a non-empty open subset $U^{\prime}$ of $U$ such that $\left(\Phi_{\beta}^{n}\right)_{\left(U^{\prime}, V\right)}$ is densely defined and lower demicontinuous on $U^{\prime}$ (by Lemma 1). Clearly, $\left\{\left(U^{\prime},\left(\Phi_{\beta}^{n}\right)_{\left(U^{\prime}, V\right)}\right)\right\} \notin \mathcal{F}^{n+1}$ and $\left\{\left(U^{\prime},\left(\Phi_{\beta}^{n}\right)_{\left(U^{\prime}, V\right)}\right)\right\} \cup \mathcal{F}^{n+1}$ is a family satisfying the properties $\left(a_{n+1}\right),\left(c_{n+1}\right)$ and $\left(d_{n+1}\right)$. This contradicts the maximality of $\mathcal{F}^{n+1}$ and hence we may conclude that $\mathcal{F}^{n+1}$ satisfies property $\left(b_{n+1}\right)$. This completes the inductive step.

Let $X_{1}:=\bigcap_{n=1}^{\infty} W^{n}$. Clearly $X_{1}$ is a dense- $G_{\delta}$ subset of $X$ and for each $x \in X_{1}$ and $n \in \mathbb{N}$ there exists a unique $\alpha_{n}(x) \in \Lambda^{n}$ such that $x \in U_{\alpha_{n}(x)}^{n}$. Therefore we can define a set-valued mapping $\Psi: X_{1} \rightarrow 2^{Y}$ by,

$$
\Psi(x):=\bigcap_{n=1}^{\infty} \overline{\Phi_{\alpha_{n}(x)}^{n}\left(U_{\alpha_{n}(x)}^{n}\right)} .
$$

Clearly, $\Psi$ is non-empty and compact-valued since for each $x \in X_{1}$,

$$
\mathcal{F}(x):=\left\{\Phi_{\alpha_{n}(x)}^{n}\left(U_{\alpha_{n}(x)}^{n}\right): n \in \mathbb{N}\right\}
$$

is a $d$-Cauchy filter-base on $Y$. So to show that $\Psi$ is an usco, it remains to show that $\Psi$ is upper semicontinuous. To this end, consider $x \in X_{1}$ and $O$ an open set containing $\Psi(x)$. Since $\Psi(x)$ is compact it will suffice to show that there exists an open neighbourhood $U$ of $x$ such that $\Psi(U) \subseteq \bar{O}$. We claim that for some $n_{0} \in \mathbb{N}, \Phi_{\alpha_{n_{0}}(x)}^{n_{0}}\left(U_{\alpha_{n_{0}}(x)}^{n_{0}}\right) \subseteq O$, for otherwise, $\mathcal{F}^{*}(x):=\left\{\Phi_{\alpha_{n}(x)}^{n}\left(U_{\alpha_{n}(x)}^{n}\right) \backslash O: n \in \mathbb{N}\right\}$ would be a $d$-Cauchy filter-base on $Y$ which would have a cluster point in $Y \backslash O$. But this is impossible since,

$$
\emptyset \neq \bigcap_{\bar{F} \in \mathcal{F} \cdot} \bar{F} \subseteq \bigcap_{\bar{F} \in \mathcal{F}} \bar{F}=\Psi(x) \subseteq O .
$$

Therefore there is some $n_{0} \in \mathbb{N}$ such that $\Phi_{\alpha_{n_{0}}(x)}^{n_{0}}\left(U_{\alpha_{n_{0}}(x)}^{n_{0}}\right) \subseteq O$ and so,

$$
\Psi(y)=\bigcap_{n=1}^{\infty} \overline{\Phi_{\alpha_{n}(y)}^{n}\left(U_{\alpha_{n}(y)}^{n}\right)} \subseteq \overline{\Phi_{\alpha_{n_{0}}(y)}^{n_{0}}\left(U_{\alpha_{n_{0}}(y)}^{n_{0}}\right)}=\overline{\Phi_{\alpha_{n_{0}}(x)}^{n_{0}}\left(U_{\alpha_{n_{0}}(x)}^{n_{0}}\right)} \subseteq \bar{O}
$$

for all $y \in U_{a_{n_{0}}(x)}^{n_{0}} \cap \cdot X_{1}$.

We now define the mapping $G: X_{1} \rightarrow 2^{Y}$ by, $G(x):=\Psi(x) \cap \Phi^{*}(x)$ for all $x \in X_{1}$. We claim that the mapping $G$ is an usco. Obviously $G$ has a closed graph as both $\Psi$ and $\Phi^{*}$ have closed graphs. Moreover, as $\operatorname{Gr}(G) \subseteq \operatorname{Gr}(\Psi)$ and $\Psi$ is an usco, we have that $G$ is also an usco (see, [1, page 309]), provided we can show that $G$ has non-empty images. So in order to obtain a contradiction, let us suppose that for some $x_{0} \in X_{1}, G\left(x_{0}\right)=\emptyset$. This means that the non-empty compact set $\left\{x_{0}\right\} \times \Psi\left(x_{0}\right)$ does not intersect the graph of 
$\Phi^{*}$. Since $\operatorname{Gr}\left(\Phi^{*}\right)$ is a closed subset of $X \times Y$, a straight forward compactness argument shows that there are open sets $U$ of $X$ and $V$ of $Y$ such that $x_{0} \in U, \Psi\left(x_{0}\right) \subseteq V$ and $(U \times V) \cap \operatorname{Gr}\left(\Phi^{*}\right)=\emptyset$. Since $\Psi\left(x_{0}\right) \subseteq V$ it follows, as above, that there exists an $n_{0} \in \mathbb{N}$ such that $\Phi_{\alpha_{n_{0}}\left(x_{0}\right)}^{n_{0}}\left(U_{\alpha_{n_{0}}\left(x_{0}\right)}^{n_{0}}\right) \subseteq V$ and so,

$$
\emptyset \neq \Phi_{\alpha_{n_{0}}\left(x_{0}\right)}^{n_{0}}\left(U_{\alpha_{n_{0}}\left(x_{0}\right)}^{n_{0}} \cap U\right) \subseteq \Phi\left(U_{\alpha_{n_{0}}\left(x_{0}\right)}^{n_{0}} \cap U\right) \cap V \subseteq \Phi^{*}(U) \cap V=\emptyset .
$$

This gives us the desired contradiction. Therefore $G$ is an usco selection of $\Phi^{*}$.

REMARK 1. In Theorem $1, \Phi^{*}$ is the unique mapping whose graph is the closure of the graph of $\Phi$ in $X \times Y$ (endowed with the product topology). In particular, if $\Phi$ has a closed graph then $\Phi^{*}=\Phi$.

An usco mapping $\Phi$ from a topological space $X$ into subsets of a topological space $Y$ is called a minimal usco if its graph does not strictly contain the graph of any other usco defined on $X$. We say that a topological space $Y$ belongs to Stegall's class if for every Baire space $X$ and minimal usco mapping $\Phi: X \rightarrow 2^{Y}, \Phi$ is single-valued at the points of a residual subset of $X$. A topological space $Y$ belongs to the class of weakly Stegall spaces if for every $\alpha$-favourable space $X$ and minimal usco mapping $\Phi: X \rightarrow 2^{Y}$, $\Phi$ is single-valued at the points of an everywhere second category subset of $X$. For the sake of completeness we recall the definition of $\alpha$-favourability.

Let $X$ be a topological space. On $X$ we consider the Banach-Mazur game played between two players $\alpha$ and $\beta$. A play of the game is a decreasing sequence of, alternately chosen, non-empty open subsets $A_{n} \subseteq B_{n} \subseteq \ldots B_{2} \subseteq A_{1} \subseteq B_{1}$, where the sets $A_{n}$ are chosen by player $\alpha$ and the sets $B_{n}$ by player $\beta$. Player $\alpha$ is said to have won a play of the game if $\bigcap_{n \in \mathbb{N}} A_{n} \neq \emptyset$. Otherwise player $\beta$ is said to have won the play. A strategy $s$ for player $\alpha$ is a rule that tells him or her how to play (possibly depending on all the previous moves of player $\beta$ ). Since the moves of player $\alpha$ may depend on the moves of player $\beta$, we denote the $n$th move of player $\alpha$ by, $s\left(B_{1}, B_{2}, \ldots, B_{n}\right)$. We say that $s$ is a winning strategy, if using it, player $\alpha$ wins every play, independently of the moves of player $\beta$. More information on Banach-Mazur game can be found in [6].

Corollary 1. Let $X$ be a Baire (an $\alpha$-favourable) space and $Y$ be a partition complete space that lies in Stegall's class (the class of weakly Stegall spaces). Suppose that $\Phi: X \rightarrow 2^{Y}$ is a densely defined lower demicontinuous mapping with closed graph. Then there exist a residual (everywhere second category) set $X_{1} \subseteq X$ and a continuous selection $\sigma: X_{1} \rightarrow Y$ of $\Phi$ on $X_{1}$.

Proof: First we shall consider the case when $X$ is a Baire space, $Y$ is partition complete and in Stegall's class. From Theorem 1 there exists an usco mapping $G: R \rightarrow 2^{Y}$ acting from a residual subset $R$ of $X$ into $Y$ such that $G(x) \subseteq \Phi(x)$ for all $x \in R$. As every usco mapping contains a minimal usco mapping (see, [2, page 649]), the mapping $G$ contains a minimal usco mapping $S: R \rightarrow 2^{Y}$. Now since the range space $Y$ belongs 
to Stegall's class the mapping $S$ is single-valued on a residual subset $X_{1} \subseteq R$. The restriction of the mapping $S$ to the set $X_{1}$ gives rise to the desired selection of $\Phi$ on $X_{1}$. In the case when the space $Y$ belongs to the class of weakly Stegall spaces and $X$ is $\alpha$-favourable the proof follows in a similar fashion except that one requires the additional fact that a residual subset of an $\alpha$-favourable space is again $\alpha$-favourable.

\section{Applications}

We say that a mapping $f: X \rightarrow Y$ from a topological space $X$ into a topological space $Y$ is demi-open if for every open set $U$ in $X$ the set int $\overline{f(U)}$ is dense in $\overline{f(U)}$. It is easy to verify that $f^{-1}: Y \rightarrow 2^{X}$ is lower demicontinuous on $Y$ if the mapping $f: X \rightarrow Y$ is demi-open on $X$.

Corollary 2. Let $f: X \rightarrow Y$ be a demi-open mapping with closed graph acting from a partition complete space $X$ which lies in Stegall's class (the class of weakly Stegall spaces) into a dense subset of a Baire space (an $\alpha$-favourable space) $Y$. Then there exists a continuous mapping $\sigma$ from a residual (everywhere second category) subset $Y_{1} \subseteq Y$ into $X$ such that $(f \circ \sigma)(x)=x$ for all $x$ in $Y_{1}$.

ProOF: Let us consider the inverse mapping $f^{-1}: Y \rightarrow 2^{X}$. This is a densely defined lower demicontinuous mapping with closed graph. Hence from Corollary 1 , there exist a residual (everywhere second category) subset $Y_{1} \subseteq Y$ and a continuous selection $\sigma: Y_{1} \rightarrow X$ of $f^{-1}$ on $Y_{1}$. It follows then that $(f \circ \sigma)(x)=x$ for all $x \in Y_{1}$.

Corollary 3. Let $h: G \rightarrow K$ be a homomorphism acting from a partition complete group $G$ into a Baire topological group $K$. If $h$ is demi-open, has a closed graph and dense range then the mapping is open and onto $K$.

Proof: The inverse mapping $h^{-1}: K \rightarrow 2^{G}$ is densely defined and lower demicontinuous with closed graph. Hence by Theorem 1 the domain of $h^{-1}$ is residual in $K$, that is, the range of $h$ is residual in $K$. However, as $h(G)$ is a subgroup of $K$ it must be the case that $h(G)=K$. To show that $h$ is open it suffices to show that for each non-empty open set $U$ in $G, h(U)$ is somewhere residual in $K$ and this follows by applying Theorem 1 to the inverse of the restriction of $h$ to $U$.

\section{REFERENCES}

[1] J.M. Borwein and W.B. Moors, 'Essentially smooth Lipschitz functions', J. Funct. Anal. 149 (1997), 305-351.

[2] J.P.R. Christensen, 'Theorems of Namioka and R.E. Johnson type for upper semicontinuous and compact valued set-valued mappings', Proc. Amer. Math. Soc. 86 (1982), 649-655.

[3] M.M. Coban, P.S. Kenderov and J.P. Revalski, 'Densely defined selections of multivalued mappings', Trans. Amer. Math. Soc. 344 (1994), 203-223. 
[4] J.R. Giles and W.B. Moors, 'A selection theorem for quasi-lower semicontinuous set-valued mappings', J. Nonlinear Convex Anal 2 (2001), 345-350.

[5] E. Micheal, 'Almost complete spaces, hypercomplete spaces and related mapping theorems', Topology Appl. 41 (1991), 113-130.

[6] J.C. Oxtoby, Measure and category, Graduate Text in Maths. 2 (Springer-Verlag, New York, 1971).

Department of Mathematics

The University of Waikato

Private Bag 3105

Hamilton

New Zealand

e-mail: moors@math.waikato.ac.nz

ss15@math.waikato.ac.nz 